\title{
Dynamical Atmospheres and Mass Loss of Pulsating AGB Stars
}

\author{
Susanne Höfner
}

Dept. of Astronomy and Space Physics, Uppsala University, Sweden

Rita Loidl

Institute f. Astronomy, University of Vienna, Austria

Bernhard Aringer, Uffe G. Jørgensen

Astronomical Observatory, Copenhagen University, Denmark

\begin{abstract}
We present a new generation of dynamic model atmospheres for pulsating AGB stars based on a combined solution of hydrodynamics, frequency-dependent radiative transfer, and time-dependent dust formation. At present, we are performing a systematic study of the dependence of atmospheric structure, observable properties and mass loss on stellar parameters and pulsation properties. Preliminary results show a good agreement with observations and a considerable improvement compared to existing grey dynamical models.
\end{abstract}

\section{Introduction}

Large-amplitude pulsations occuring in asymptotic giant branch stars (e.g. Mira variables) both affect the spectra of these stars and play an important role for their mass loss by stellar winds. The pulsation triggers the formation of strong, radiating shock waves which propagate outwards through the stellar atmosphere, modifying its structure both locally and globally. The resulting levitation of the outer atmospheric layers can lead to efficient formation of molecules and dust grains, causing a stellar wind driven by radiation pressure (see Sedlmayr, these proceedings).

The detailed self-consistent modelling of the highly dynamical atmospheres is crucial for a reliable interpretation of the observational data on these pulsating stars. Furthermore, the prediction of mass loss rates from first principles is essential for understanding stellar evolution in general and the AGB phase in particular, since mass loss becomes the dominant phenomenon during this late evolutionary phase of low and intermediate mass stars.

\section{Modelling Method}

We have created a new generation of dynamical model atmospheres for pulsating AGB stars by combining time-dependent hydrodynamics with frequency- 
dependent radiative transfer, and a detailed description of dust formation (moment method, for carbon grains; Gail \& Sedlmayr, 1988; Gauger et al., 1990).

The variable structure of the atmosphere is obtained by solving the equations of hydrodynamics together with the frequency-integrated zeroth and first moment equations of the radiative transfer equation, describing the momentum and energy balance of the radiation field. The frequency-integrated opacities and the Eddington factor, which are required to close the system of conservation equations, result from a solution of the frequency-dependent radiative transfer equation for the given density-temperature structure at each time step. The models presented here are based on opacity sampling data of molecular opacities at 51 randomly chosen frequency points. For a more detailed description of the modelling method, see Höfner (1999).

The dynamical computation starts with a dust-free hydrostatic initial model, which is characterized by the following parameiers: luminosity $L_{*}$, effective temperature $T_{*}$, mass $M_{*}$ and the elemental abundances. The stellar pulsation is simulated by applying a variable inner boundary $R_{\text {in }}(t)$ below the photosphere which is moving sinusoidally with a velocity amplitude $\Delta u_{p}$ and a period $P$. Since the total flux is kept constant there the luminosity at the inner boundary varies proportional to $R_{\mathrm{in}}^{2}$ (see Höfner \& Dorfi, 1997 for details).

\section{Results and Conclusions}

To demonstrate the influence of pulsation on the structure of the atmosphere we have chosen two dynamical models, which start from the same hydrostatic initial model with the following parameters: luminosity $L_{*}=7000 L_{\odot}$, effective temperature $T_{*}=2800 \mathrm{~K}$, mass $M_{*}=1 M_{\odot}$ and a carbon-to-oxygen ratio of 1.4 . The pulsation period is $P=390 \mathrm{~d}$ and we use two different velocity amplitudes for the inner boundary, i.e. $\Delta u_{p}=2 \mathrm{~km} \mathrm{~s}^{-1}$ and $\Delta u_{p}=4 \mathrm{~km} \mathrm{~s}^{-1}$. The resulting spatial structures of velocity, density, temperature and degree of condensation at different phases are shown in Fig. 1.

The model with the smaller pulsation amplitude (shown in the left column) does not develop a stellar wind: the dynamic levitation of the outer layers of the atmosphere is not sufficient to allow the formation of dust grains. However, if a larger pulsation amplitude is applied to the same initial model (right column) the outer atmospheric layers are cool and dense enough to provide an environment for efficient dust formation. The dust formation cycle in this model spans two pulsation periods: every other period a dust shell is created and the spatial pattern of the model repeats itself on this time scale. The radiation pressure on the grains drives a stellar wind with a velocity of $11 \mathrm{~km} \mathrm{~s}^{-1}$ and a mass loss rate of about $2 \cdot 10^{-6} M_{\odot} \mathrm{yr}^{-1}$, and the structure of the atmosphere as well as the resulting spectra are drastically different form the first model. Note however that even in the model with the lower pulsation amplitude (which has no wind) the atmospheric structure is strongly affected by the shock waves and cannot be modelled by a sequence of hydrostatic structures with different luminosities and effective temperatures.

At present, we are systematically investigating the dependence of observable properties (spectra at different resolutions, photometric colours, radii, etc.) on pulsation characteristics, phase, and stellar parameters. In contrast to the 

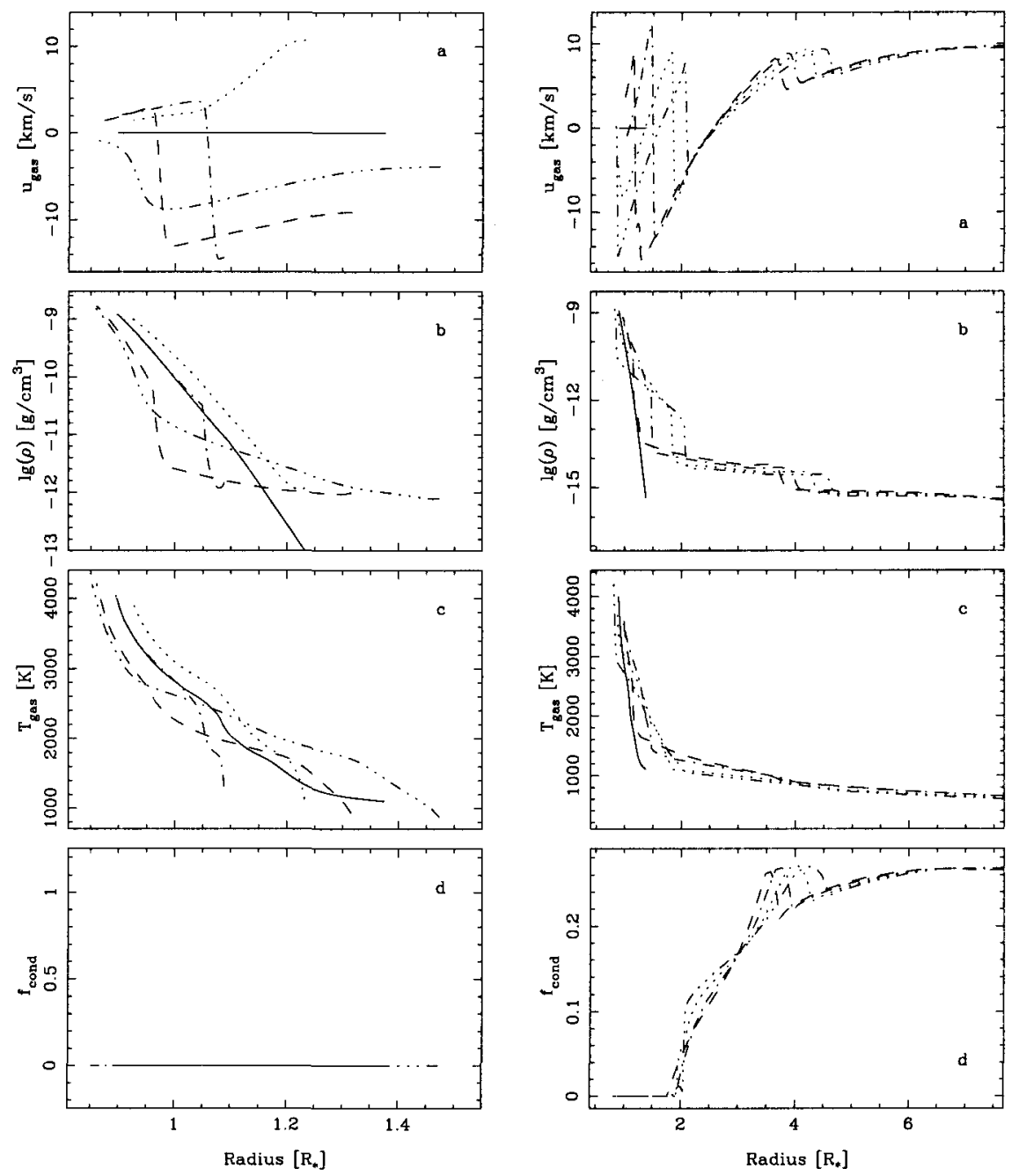

Figure 1. Radial structure of two dynamical models: (a) velocity, (b) gas density, (c) gas temperature and (d) degree of condensation as a function of the radius in units of the stellar radius $R_{*}$. The left column shows a model with a piston velocity $\Delta u_{p}=2 \mathrm{~km} \mathrm{~s}^{-1}$ at 4 different phases (dashed, dash-dotted, dotted and dash-tripple-dotted lines), the right column a model with $\Delta u_{p}=4 \mathrm{~km} \mathrm{~s}^{-1}$ but otherwise identical parameters (see text). The solid curves in both columns represent the (identical) hydrostatic initial model. Note the difference in radial scales and the fact that only the model with the stronger pulsation (shown in the right column) develops a dust-driven stellar wind. 
existing grey models our new calculations properly take into account the crucial effects of molecular opacities on the atmospheric structure, thus leading to much more realistic models which are comparable to classic model atmospheres in the hydrostatic limit. Prototypes of these new models show good agreement with various observational results, and in some cases even reproduce features which cannot be explained by classical hydrostatic model atmospheres (e.g. Höfner et al., 2000; Aringer et al., 2000; Loidl et al., 2000; Aringer et al. and Andersen et al., these proceedings).

The mass loss rates and wind velocities resulting from our models for Crich stars show good agreement with existing observations. In this context, we stress the importance of both, consistent microphysical data of the dust grains (see Andersen et al., this volume), and realistic atmospheric models for reliable predictions of mass loss rates.

Acknowledgments. This work has been supported by the Swedish Research Council and the Royal Swedish Academy of Sciences.

\section{References}

Aringer, B., Kerschbaum, F., Hron, J., \& Höfner, S. 2000, in ISO beyond the Peaks: The 2nd ISO workshop on analytical spectroscopy, eds. A. Salama A., M. Kessler, K. Leech, \& B. Schulz, (ESA SP-456), 295

Gail, H.-P. \& Sedlmayr, E. 1988, A\&A, 206, 153

Gauger, A., Gail, H.-P., Sedlmayr, E. 1990, A\&A, 235, 345

Höfner, S. 1999, A\&A, 346, L9

Höfner, S. \& Dorfi, E.A. 1997, A\&A, 319, 648

Höfner, S. Loidl, R., Aringer, B., et al. 2000, in ISO beyond the Peaks: The 2nd ISO workshop on analytical spectroscopy, eds. A. Salama A., M. Kessler, K. Leech, \& B. Schulz, (ESA SP-456), 299

Loidl, R., Hron, J., Jørgensen, U.G., \& Höfner, S. 2000, in ISO beyond the Peaks: The 2nd ISO workshop on analytical spectroscopy, eds. A. Salama A., M. Kessler, K. Leech, \& B. Schulz, (ESA SP-456), 315

\section{Discussion}

S. Owocki : Is your code Eulerian or Lagrangian? Also, in applying your variable base speed from pulsation at the lower boundary, how do you self-consistently allow mass flux through the boundary, which is required for the wind outflow?

S. Höfner: We are using an adaptive grid which is neither Eulerian nor Lagrangian. The location of the grid points is determined by an equation, which is solved simultaneously with the physical equations. The variable inner boundary acts like a pulsating membrane. No mass flux is allowed across this boundary which is located below the stellar photospere. The fraction of the stellar mass contained in the computational domain is usually large enough for the effects of mass loss to be negligible during the model calculation. 